\title{
La hipótesis de eficiencia y la modelación de series bursátiles mexicanas: un analisis multivariado
}

The efficient-market hypotesis and the modelling of mexican stock series: a multivariate analysis

Antonio Ruiz-Porras* y Brenda Ruiz-Robles**

\section{Resumen}

Estudiamos la Hipótesis de Eficiencia de los Mercados (EMH) y modelamos las series de rendimientos bursátiles mexicanos. El estudio utiliza pruebas de raíces unitarias y doce modelos GARCH multivariados. Los principales resultados sugieren que: 1) El mercado bursátil mexicano es ineficiente en forma débil; 2) la eficiencia ha disminuido desde el año 2007; 3) los modelos de Correlación Condicionada Constante (CCC) son los que mejor describen los rendimientos; 4) las buenas y malas noticias tienen impactos asimétricos sobre la volatilidad; y 5) las perturbaciones parecen seguir una distribución $t$ de Student multivariada. El estudio usa series diarias de los precios de 20 acciones y del Índice de Precios y Cotizaciones (IPC) para el periodo 02/10/2000-10/10/2012.

\section{Palabras clave:}

- Hipótesis de Eficiencia de Mercados

- Modelos garch Multivariados

- Series Bursátiles

- Crisis Financiera Global

\section{Abstract}

We study the Efficient-Market Hypothesis (ЕмH) and we model the series of Mexican stock returns. The study uses unit-root tests and twelve multivariate GARCH models. The main results suggest that: 1) The Mexican stock market is weakly inefficient; 2) efficiency has declined since 2007; 3) Constant Conditional Correlation (CCC) models are best ones that describe the returns; 4) good and bad news have asymmetric impacts on volatility; and 5) perturbations seem to follow a multivariate Student's t distribution. The study uses 20 daily series of stock prices and of the Mexican Stock-Market Index (IPC) for the period 02/10/2000$10 / 10 / 2012$.

\section{Keywords: \\ - Efficient-Market Hypothesis \\ - Multivariate GARCH models \\ - Stock Series \\ - Global Financial Crisis}

JEL: C58, C32, G14

\section{Introducción}

Tradicionalmente se considera que los mercados bursátiles son importantes medios de desarrollo económico y financiero. En la literatura se sustenta dicha importancia con base en la consideración de que los mercados bursátiles cumplen funciones necesarias para el buen desempeño de las economías. ${ }^{1}$ Entre otras, estas funciones incluyen: 1) la provisión liquidez para financiar proyectos; 2) la diversificación de riesgos; 3) la provisión de información de las empresas; 4) el control de malas prácticas de gobierno corporativo; y 5) la movilización de ahorros. Usualmente se considera que el cumplimiento de estas funciones depende en gran medida de la eficiencia de los mercados bursátiles.

\footnotetext{
${ }^{1}$ Una de las revisiones clásicas de la literatura sobre cómo los mercados bursátiles fomentan el desarrollo económico es el trabajo de Levine y Zervos (1996).

* Email: antoniop@cucea.udg.mx Dirección: Departamento de Métodos Cuantitativos. Universidad de Guadalajara, CuCEA. Periferico Norte 799, Núcleo Universitario Los Belenes, 45100, Zapopan, Jalisco, México.

** Email: brenda_ruiz2587@yahoo.com.mx Dirección: Calzada Circunvalación Poniente núm. 49, Ciudad Granja, 45010, Zapopan, Jalisco, México.
} 
Paradójicamente, el término de "eficiencia del mercado bursátil” es relativamente reciente y sujeto a controversias en la economía financiera. Eugene Fama introduce el término para hacer referencia a aquellos mercados en donde los precios de los activos reflejan plenamente la información disponible (Fama, 1965). Más aun, Fama define una teoría alrededor de dicho término. La Hipótesis de Eficiencia de los Mercados (Efficient-Market Hypothesis, EMH), señala que no es posible obtener consistentemente rendimientos financieros superiores a aquellos del promedio del mercado. Asimismo, postula la imposibilidad de describir los precios de los activos usando modelos estadísticos.

En las literaturas teórica y empírica existen agudas controversias sobre la validez de la hipótesis de eficiencia de los mercados bursátiles. Las revisiones de estudios no ofrecen evidencia concluyente en ningún sentido. Los hallazgos empíricos relativos a la EMH dependen en buena medida del nivel de desarrollo, de los periodos considerados y de las economías analizadas. Las posiciones entre quienes argumentan en favor y en contra de la validez de la EMH se han polarizado tras la crisis financiera global. Por una parte, están quienes atribuyen esta crisis a la suposición de validez de la EMH. Por otra, están quienes sustentan que la EMH ha sido entendida de una manera muy restrictiva. ${ }^{2}$

En esta investigación se estudia la validez de la hipótesis de eficiencia en el mercado bursátil mexicano y se modelan sus series de rendimientos bursátiles. La evaluación de la EMH, en su forma débil, se realiza mediante estadísticas descriptivas, pruebas de raíces unitarias y de correlación. Los ejercicios de modelación y análisis econométricos utilizan doce modelos GARCH multivariados con fines de comparativos y de caracterización de las series durante periodos de calma e inestabilidad financiera. El estudio se sustenta en una muestra de series de precios diarios de veinte acciones y del Índice de Precios y Cotizaciones (IPC) que abarca datos del 2 de octubre de 2000 al 10 de octubre de 2012.

Académicamente, la investigación complemente la literatura referida a la evaluación de la eficiencia del mercado bursátil mexicano; y la literatura referida a la modelación de series con modelos GARCH multivariados. Particularmente, a diferencia de otros estudios, aquí se utilizan pruebas de estacionariedad y de correlación para evaluar la EMH. Asimismo, se modelan y analizan las series de rendimientos bursátiles, de manera conjunta, mediante diversos modelos GARCH multivariados. En este contexto, vale la pena señalar que se analizan, modelan y comparan las series bursátiles considerando periodos de relativa "calma" e "inestabilidad" en los mercados internacionales.

${ }^{2}$ Véase Ball (2009) para una revisión de los argumentos que sustentan ambas posiciones. 
El estudio está organizado en siete secciones. La Sección 2 incluye la revisión de la literatura. En la Sección 3 se describe la metodología de análisis. La Sección 4 describe la base de datos y muestra la estadística descriptiva de las series de rendimientos. La Sección 5 muestra los resultados del análisis de estacionariedad. La Sección 6 muestra los ejercicios de modelación y análisis econométricos. Asimismo, desarrolla el análisis comparativo. La Sección 7 sintetiza los resultados y menciona algunas líneas de investigación futura.

\section{Revisión de la literatura}

En esta sección se revisan los estudios referentes a la hipótesis de la eficiencia de los mercados, los modelos GARCH multivariados y el concepto de estacionariedad. Por esta razón, la sección se divide en dos apartados. En el primer apartado se explica la Hipótesis de Eficiencia de los Mercados (EMH), sus implicaciones y las controversias que la misma ha planteado. En el segundo se describen las estructuras de los modelos GARCH multivariados y se analiza la vinculación entre la EMH y la estacionariedad de las series de tiempo. En ambos apartados se hace énfasis en los estudios empíricos realizados en el contexto mexicano. Esto con fines de contextualizar y justificar el estudio desarrollado.

\section{I La Hipótesis de Eficiencia de los Mercados}

En la economía y las finanzas existen varias teorías que explican el comportamiento de los mercados y las series bursátiles. Probablemente la más conocida es la Hipótesis de Eficiencia de los Mercados (EMH) propuesta por Fama (1965). ${ }^{3}$ Esta teoría postula que, en un mercado "eficiente", los precios de los activos reflejan plenamente la información disponible en dicho mercado. ${ }^{4}$ Más aun, postula la imposibilidad de describir y predecir los precios de los activos

3 Tradicionalmente se le atribuye a Fama (1965) la propuesta de la teoría de la eficiencia de mercado. Sin embargo, parece que en realidad la misma fue propuesta por Louis Bachelier bajo el nombre de la teoría del "estado estático del mercado" (Bachelier, 1900). Hyme (2003) atribuye el cambio de terminología y autoría de la teoría a los economistas denominados como Nuevos Clásicos. Estos economistas, en contraposición de los keynesianos, consideran que el equilibrio walrasiano es permanente y que las expectativas de los agentes económicos y financieros se determinan mediante procesos racionales.

${ }^{4}$ Adviértase que la definición de eficiencia es de tipo informacional y no de tipo distributivo. En el contexto informacional, la eficiencia se refiere a la manera en que los precios de los activos reflejan la información existente. En el contexto distributivo, la eficiencia se relaciona con la asignación óptima de recursos escasos entre los agentes de la economía.Véase Cortés y Corzo (2009) para una discusión y análisis sobre los conceptos de eficiencia informacional y distributiva. 
de manera sistemática usando modelos estadísticos. Por tanto, si un mercado es eficiente, el mejor pronóstico para el precio de mañana de un activo cualquiera será su precio del día de hoy.

Empíricamente, la hipótesis de eficiencia tiene importantes implicaciones econométricas. ${ }^{5}$ Particularmente, si se considera que su cumplimiento conlleva a la imposibilidad de que los agentes puedan obtener ganancias sistemáticas por hacer transacciones bursátiles; la EMH implica que el valor esperado de los rendimientos debiera ser cero. La EMH también implica que los rendimientos no pueden estar correlacionados en el tiempo y que los modelos estadísticos carecen de utilidad práctica. Esto ocurre debido a que la EMH explica los movimientos de los precios como procesos de caminata aleatoria (Fama, 1965); o, más generalmente, como martingalas (Samuelson, 1965; Mandelbrot, 1966).

En la literatura empírica existen varias revisiones de estudios que han evaluado la validez de la EMH en los mercados bursátiles. Sin embargo, las revisiones no ofrecen evidencia concluyente en ningún sentido. Aparentemente, los resultados reportados dependen del nivel de desarrollo, de los periodos considerados y de las economías analizadas. Entre las revisiones que reportan evidencia en favor de la EMH están las de Fama (1970) y Malkiel (2003) y (2005). Entre las revisiones que hallan evidencia en contra están las de Shleifer (2001) y Timmermann y Granger (2004). Entre las revisiones que ofrecen evidencia mixta se encuentran las de Hyme (2003), Yen y Lee (2008) y Duarte y Mascareñas (2013).

Empíricamente, la falta de evidencia concluyente puede atribuirse, entre otros factores, a que no existe una definición única de la EMH. La literatura especializada distingue entre las formas de eficiencia débil, semi-fuerte y fuerte. Particularmente, la forma débil establece que los precios presentes incorporan toda la información de los precios pasados (Aragonés y Mascareñas, 1994). La forma semi-fuerte establece que los precios incorporan toda la información hecha pública que afecte el valor de los activos. La forma fuerte establece que los precios incorporan toda la información pública y privada que se puede conocer (Mehrara y Oryoie, 2012).

En México, existen algunos estudios que han analizado la hipótesis de eficiencia en el mercado bursátil en su forma débil. Particularmente, LópezHerrera (1998) argumenta en contra de la misma mediante un análisis los precios del Índice de Precios y Cotizaciones (IPC) entre 1987 y 1997. El estudio se sustenta en regresiones de mínimos cuadrados ordinarios (MCO-OLS) y en tests de autocorrelación. Santillán-Salgado (2011) argumenta en favor de la EMH ${ }^{5}$ Véase Uribe-Gil y Ulloa-Villegas (2011) para una derivación matemática de estas implicaciones. 
mediante un análisis de los rendimientos, a nivel agregado y desagregado, entre 2000 y 2010. El estudio también se sustenta en regresiones MCO-OLs. Por tanto, los estudios referidos al mercado mexicano también proveen resultados mixtos. ${ }^{6}$

Metodológicamente, los estudios realizados muestran que existen áreas de oportunidad para el desarrollo de investigación en el contexto del mercado bursátil mexicano. Los estudios existentes, en su mayoría, asumen que se cumplen los supuestos del modelo de regresión lineal clásico de mínimos cuadrados (MCO-OLS). Sin embargo, es posible argumentar que las series bursátiles suelen mostrar comportamientos no lineales, autorregresivos y heteroscedásticos que no son compatibles con los supuestos de MCO-OLs. Además, la teoría sugiere que las evaluaciones de la EMH deben centrarse en los precios de los activos bursátiles y en sus rendimientos, tanto a nivel agregado como desagregado.

Los comentarios señalados muestran que es necesario hacer investigación relativa a la validez de la EMH en el mercado bursátil mexicano y a la modelación de series de rendimientos. El mercado bursátil mexicano refleja la confianza y las expectativas de los agentes en la economía. Muchas decisiones de financiamiento empresarial, de ahorro e inversión y de administración de riesgos dependen del comportamiento y de la volatilidad de este mercado. Por esta razón, los ejercicios de modelación y análisis econométricos resultan pertinentes y complementarios a la evaluación de la EMH. De hecho, la investigación aquí planteada se sustenta en estas consideraciones.

\subsection{Modelación econométrica de series bursátiles y la estacionariedad de las series}

La modelación econométrica de series bursátiles ha tenido gran desarrollo en las últimas décadas. Particularmente, los modelos de la familia ARCH ha sido los más usados para describir y analizar dichas series. Estos modelos asumen la existencia de procesos autorregresivos con heteroscedasticidad condicionada (AutoRegressive Conditional Heteroskedasticity, ARCH). Los modelos ARCH permiten describir series con comportamientos caracterizados por no linealidades, volatilidades excesivas clusters de volatilidad, volatilidades no constantes y

\footnotetext{
${ }^{6}$ Un estudio reciente relacionado es el de Cabrero, Cermeño y Hernández-Trillo (2013). Ellos argumentan a favor de la EMH mediante un análisis bootstrap del tipo de cambio peso-dólar entre 1997 y 2011.
} 
distribuciones no normales. ${ }^{7}$ Los primeros modelos de esta familia fueron desarrollados por Engle (1982) y Bollerslev (1986).

Los modelos de la familia ARCH se dividen en univariados y multivariados. Los modelos univariados modelan la media y la varianza de series individuales. Los modelos multivariados lo hacen para conjuntos de series. En la literatura empírica se usan modelos univariados con fines de descripción y análisis de series financieras y bursátiles. ${ }^{8}$ Paradójicamente, es reconocido que los mercados y activos financieros suelen moverse de manera conjunta a lo largo del tiempo. Sin embargo, los trabajos que usan modelos multivariados son relativamente escasos. ${ }^{9}$ Esta situación se justifica, entre otras razones, porque estos modelos requieren la estimación de un gran número de parámetros.

Los modelos GARCH multivariados asumen diversos supuestos sobre la dinámica conjunta de las series económicas y financieras. Estas dinámicas se postulan mediante supuestos relativos al vector de la media condicional, de la matriz de varianza-covarianza condicional y de la distribución de densidad multivariada de las perturbaciones. Particularmente, las matrices de varianzacovarianza son importantes porque las mismas definen la estructura dinámica de los modelos multivariados. En la literatura especializada, las matrices de varianza-covarianza más usadas son de los tipos DVECH (Diagonal VECH), DBEKK (Diagonal BEKK) y CCC (Constant Conditional Correlation).

Los modelos GARCH multivariados (MGARCH, Multivariate GARCH) muy pocas veces se han usado para analizar y modelar series económicas y financieras mexicanas. La mayoría de los estudios publicados modelan y analizan los rendimientos bursátiles a nivel agregado y su relación con las dinámicas de otras series internacionales. ${ }^{10}$ Metodológicamente, estos estudios suelen asumir especificaciones de tipo GARCH sin efectos apalancamiento. Entre los estudios que han usado modelos mararch destacan los de Ludlow y Mota (2006), Domínguez (2006), López-Herrera, Ortiz y Cabello (2009) y Lorenzo-Valdés, Durán-Vázquez y Armenta-Fraire (2012).

\footnotetext{
${ }^{7}$ Adviértase que ninguno de estos comportamientos es susceptible de ser modelado mediante el modelo de regresión lineal clásico de mínimos cuadrados ordinarios.

${ }^{8}$ Véase el estudio de Lorenzo-Valdés y Ruíz-Porras (2012) para revisiones de las aplicaciones de los modelos GARCH univariados para describir el comportamiento de series financieras y bursátiles mexicanas.

${ }^{9}$ Véase los trabajos de Laurent y Rombouts (2006) y Minovic (2009) para revisiones en el contexto de economías desarrolladas de las aplicaciones de los modelos GARCH multivariados.

${ }^{10}$ Los trabajos de López-Herrera y Rodríguez-Benavides (2010) y López-Herrera,Venegas-Martínez y Gurrola-Ríos (2012) constituyen excepciones. El primer trabajo analiza las relaciones del mercado accionario mexicano con el crecimiento económico. El segundo analiza las relaciones del IPC, el tipo de cambio, el $\mathrm{EMBI}+\mathrm{y}$ la tasa de interés.
} 
Los estudios arriba señalados han analizado diversas temáticas bursátiles, aunque ninguno analiza la validez de la hipótesis de eficiencia a nivel agregado y desagregado. Particularmente, Ludlow y Mota (2006) estudian la dinámica conjunta de los rendimientos de los índices del IPC, Nasdaq y S\&P500. Domínguez (2007) analiza la dinámica del IPC con otros índices latinoamericanos. López-Herrera, Ortiz y Cabello (2009) estudian las relaciones entre los mercados de valores del TLCAN. Lorenzo-Valdés, Durán-Vázquez y ArmentaFraire (2012) analizan los rendimientos del IPC y los cambios en los precios del petróleo usados como referencia en el mercado internacional.

Estadísticamente, los modelos de la familia $\mathrm{ARCH}$ requieren que las series de tiempo satisfagan ciertos requisitos para que puedan usarse con fines de modelación y pronóstico. ${ }^{11}$ Estos requisitos han limitado el uso de los modelos de la familia ARCH y, en particular, de los modelos GARCH multivariados. El más importante requisito estadístico es que las series de tiempo deben ser estacionarias a fin de evitar la posibilidad de correlaciones espurias. Esto significa que la media y varianza de las series no deben variar sistemáticamente en el tiempo. Naturalmente, esto conlleva a que las series modeladas no deban comportarse como procesos de caminata aleatoria.

La validación de la hipótesis de eficiencia de los mercados (EMH) está fuertemente vinculada al concepto de estacionariedad. La EMH, en su forma débil, requiere que las series de precios bursátiles sean expresadas como procesos de caminata aleatoria. Esta consideración sugiere que la eficiencia de las series de precios bursátiles podría analizarse con base en pruebas de estacionariedad de las series. Particularmente, una condición necesaria para validar la EMH seria que las series de precios fueran no estacionarias. ${ }^{12} \mathrm{Si}$ ese fuera el caso, la validación de la EMH podría justificarse a condición de que no hubiera una correlación significativa entre los rendimientos presentes y rezagados.

Metodológicamente, hay que enfatizar que esta revisión justifica y sugiere algunos criterios para estudiar las series bursátiles mexicanas a nivel agregado y desagregado. Particularmente, el estudio se justifica debido a las transformaciones que han experimentado los mercados financieros tras la crisis global. En este contexto, la revisión sugiere que la evaluación de la hipótesis de eficiencia

\footnotetext{
${ }^{11}$ Una descripción formal de los requisitos que deben satisfacer las series de tiempo para ser modeladas se encuentra en Hamilton (1994).Véase, en particular, el capítulo 15.

${ }^{12}$ La mayoría de los trabajos que evalúan la eficiencia de los mercados llegan a una conclusión similar aunque de maneras diferentes. En lo que se refiere a los estudios realizados para el mercado bursátil mexicano, López-Herrera (1998) usa este enfoque para evaluar la validez de la EMH. Un estudio que ha analizado la estacionariedad de los precios bursátiles, aunque no en el contexto de la evaluación de hipótesis la eficiencia, es el de Durán-Vázquez, Lorenzo-Valdés y Ruíz-Porras (2012).
} 
en su forma débil podría realizarse mediante pruebas de estacionariedad y de correlación. Asimismo, sugiere que la modelación y el análisis de las series estacionarias podrían hacerse mediante modelos MGARCH y especificaciones del tipo GARCH y TGARCH.

\section{Metodología de análisis}

En esta sección se describe la metodología usada para analizar la hipótesis de eficiencia y la modelación de series bursátiles mexicanas. En este contexto debe señalarse que los activos analizados aquí son aquellos que cotizaron continuamente en la Bolsa Mexicana de Valores (BMV) entre los años 2000 y 2012. Estos activos son los que definen las series de precios y rendimientos utilizadas aquí. Particularmente, las series de rendimientos de estos activos se estiman con los precios de cierre de cada activo $i$ en el periodo $t, P_{i t}$. Así se define el rendimiento de un activo $i$ en el periodo $t, r_{i t}$, como:

$$
r_{i t}=\ln P_{i t}-\ln P_{i t-1}
$$

Metodológicamente, el estudio se sustenta en un enfoque multivariado para evaluar la hipótesis de eficiencia y la modelación de series bursátiles. Particularmente, se sustenta en análisis de estadística descriptiva, pruebas estadísticas de raíces unitarias y en el uso de modelos MGARCH. El análisis de estadística descriptiva se usa para caracterizar el comportamiento de las series de rendimientos. Las pruebas de raíz unitaria se usan para evaluar el orden de integración de las series de precios y rendimientos. Los modelos MGARCH se usan con fines de descripción y análisis de las series. Además, los modelos MGARCH y las pruebas de raíz unitaria se usan conjuntamente para evaluar la EMH.

Estadísticamente, el análisis de estacionariedad se usa para analizar la pertinencia de modelar las series bursátiles y la validez de la hipótesis de eficiencia. La modelación econométrica de series requiere que las mismas sean integradas de orden cero $\mathrm{I}(0)$ (i.e., estacionarias). Asimismo, y tal como ya se ha mencionado, la hipótesis de eficiencia requiere que las series de precios no sean estacionarias, lo cual implica que las mismas deben ser al menos de orden uno I(1). En este contexto, aquí se usa el test Aumentado de Dickey-Fuller (ADF) para analizar el orden de integración de las series bursátiles. La hipótesis nula en dicho test es que existen raíces unitarias (i.e., la serie es no-estacionaria). 
Estadísticamente, las series bursátiles no solo deben ser estacionarias para ser modeladas econométricamente. También deben manifestar efectos ARCH (i.e., los rendimientos cuadráticos deben estar autocorrelacionados).

Los modelos MGARCH se usan con fines de modelación econométrica y de análisis de las series bursátiles. Los modelos usados aquí son de los tipos DVECH (Diagonal VECH), DBEKK (Diagonal BEKK) y CCC (Constant Conditional Correlation). Estos modelos son variaciones de los propuestos, respectivamente, por Bollerslev, Engle y Wooldridge (1988), Engle y Kroner (1995) y Bollerslev (1990). Particularmente, los modelos empleados consideran especificaciones de tipo GARCH y TGARCH para analizar los posibles efectos de los shocks informacionales sobre la volatilidad de las series. Asimismo, los modelos consideran distintas distribuciones de densidad multivariada (Normal y $t$ de Student).

Los modelos MGARCH estimados permiten estudiar diversos patrones de comportamiento dinámico de las series bursátiles considerando los periodos previo y posterior a 2007. Cada modelo se construye mediante una matriz de varianza-covarianza condicionada, una especificación y una distribución de densidad multivariadas. Las matrices de varianza-covarianza condicionada, $H_{t}$, son aquellas de los modelos DVECH, DBEKK у CCC. ${ }^{13}$ Las especificaciones son la GARCH y la TGARCH. Las distribuciones son la normal y $t$ de Student multivariadas. Así, en esta investigación se usan doce modelos multivariados distintos para efectos comparativos y de estimación econométrica.

Tradicionalmente las estructuras dinámicas de los modelos MGARCH se plantean como sistemas de ecuaciones. Las tres expresiones matriciales que definen a los modelos usados en esta investigación son las siguientes:

$r_{i t}=\varphi_{i 0}+\varphi_{i 1} r_{i t-1}+\varepsilon_{i t}$

$\varepsilon_{t}=H^{\frac{1}{2}}(\theta) z_{t}$

$\operatorname{VarCov}\left(r_{t} \mid I_{t-1}\right)=H^{\frac{1}{2}} \operatorname{Var}_{t-1}\left(z_{t}\right)\left(H^{\frac{1}{2}}\right)^{\prime}=H_{t}$

, donde $r_{t}$ es un vector que incluye una serie de tiempo multivariada de $\mathrm{N}$ procesos estocásticos; $\theta$ es un vector de parámetros; $H^{\frac{1}{2}}(\theta)$ es una matriz definida positiva $N \times N$; y $z_{t}$ es un vector aleatorio $N \times 1$, tal que $E\left(z_{t}\right)=0 \mathrm{y}$ $\operatorname{Var}\left(z_{t}\right)=I_{N}$.

13 Las matrices de varianza-covarianza de los modelos DVECH, DBEKK y CCC se definen en el Apéndice. 
Metodológicamente, los ejercicios de modelación y análisis utilizan grupos de estimaciones que describen la dinámica conjunta de las series durante tres periodos específicos. Los periodos analizados corresponden a los años 20002012, 2000-2007 y 2007-2012. Así se facilita el análisis comparativo de los periodos previo y posterior a la manifestación de la crisis global. Cada grupo de estimaciones modela la dinámica conjunta de las series usando cada uno de los modelos MGARCH. Por tanto, cada grupo de estimaciones se integra por doce estimaciones distintas por periodo. Estadísticamente, todas las estimaciones se realizan usando el método de Máxima Verosimilitud (Maximum Likelihood, $M L)$.

Estadísticamente, y por consistencia con otros estudios, en todos los modelos estimados se asume que las perturbaciones siguen un proceso AR(1). Este supuesto se explicita en términos de la definición de la perturbación, $\varepsilon_{i t}$, como: $\varepsilon_{i t}=c+\rho_{i} \varepsilon_{i t}+v_{i t}$, donde $c$ es una constante y $v_{i t}$ es ruido blanco. En este contexto, la evaluación de la significancia de $\rho_{i}$ resulta un test adicional sobre la validez de la EMH. Esto en virtud de que la significancia de $\rho_{i}$ implicaría la existencia de autocorrelación, eventualmente de orden infinito, en las series de rendimientos. Por esta razón, aquí se hacen explicitas las estimaciones y los p-values asociados a las estimaciones de $\rho_{i}$.

Finalmente, el análisis comparativo y de evaluación de los modelos MGARCH se sustenta en estimadores de bondad de ajuste. Particularmente, los estimadores usados aquí son aquellos del logaritmo de verosimilitud (Log-Likelihood) y del criterio de información de Akaike (AIC). La relevancia de usar estos estimadores es que proporcionan criterios alternativos para elegir al mejor modelo descriptivo de las series cuando las matrices de varianza-covarianza son semidefinidas positivas. Más aun, proveen elementos para sugerir la existencia de algunos patrones de comportamiento dinámico conjunto de las series bursátiles mexicanas durante los periodos considerados.

\section{Base de datos y estadística descriptiva}

Este estudio usa una muestra de datos bursátiles desagregados y agregados obtenida de Infosel. La muestra incluye los precios de cierre diarios de veinte acciones individuales que cotizaron entre el 2 de octubre de 2000 y el 10 de octubre de 2012. Asimismo incluye datos diarios del Índice de Precios y Cotizaciones (IPC). Todos los datos considerados son nominales. Los activos incluidos en la muestra son aquellos para los que existen datos completos para 
el periodo. Por conveniencia estadística, las series de precios son expresadas de forma logarítmica. Así, la muestra se integra por un total de 21 series de logaritmos de precios. Cada serie incluye 3022 observaciones diarias.

La muestra analizada se caracteriza por experimentar periodos de relativa calma e inestabilidad en los mercados bursátiles internacionales. Por esta razón, las series de precios se agrupan considerando tres periodos. El primero incluye las series completas. El segundo incluye datos de las series para el periodo entre el 02 de octubre de 2000 al 9 de agosto de 2007. El tercero incluye datos para el periodo del 10 de agosto de 2007 al 10 de octubre de 2012. Los periodos utilizados se usan con fines de comparación y análisis. Así, el primer periodo se usa como referencia. El segundo refiere a una etapa de "calma" en los mercados. El tercero, por contraste, refiere a una etapa de "inestabilidad" financiera. ${ }^{14}$

El cuadro 1 muestra la estadística descriptiva de las series de rendimientos bursátiles considerando la totalidad de los datos. Esta cuadro es importante porque provee valores de referencia para evaluar la hipótesis de eficiencia. El cuadro muestra que la media estimada de rendimientos del Índice de Precios y Cotizaciones (IPC) es positiva. A nivel desagregado, el cuadro muestra que la mayoría de las medias de los activos individuales tienden a cero. El cuadro también muestra que 16 series exhiben un sesgo negativo y las restantes un sesgo positivo. Por tanto, la evidencia sugiere que las series son asimétricas. Además, el cuadro muestra todas las series son leptocúrticas y que ninguna se distribuye normalmente.

\footnotetext{
14 Los días 9 y 10 de agosto de 2007 se coordinaron la FED, el Banco de Canadá, el Banco Central Europeo, el Banco de Japón y el Banco de Reservas de Australia para estabilizar la caída generalizada de los mercados bursátiles internacionales. Este evento fue, muy probablemente, la primera gran manifestación de la crisis financiera global.
} 


\section{Cuadro |}

\section{Estadística descriptiva de los rendimientos de los activos para el periodo del 2 de octubre de 2000 al 10 de octubre de 2012 y prueba de normalidad de Jarque-Bera}

\begin{tabular}{|l|r|r|r|r|r|r|}
\hline \multicolumn{1}{|c|}{ Activo Bursátil } & Media & $\begin{array}{c}\text { Desv. } \\
\text { Est. }\end{array}$ & $\begin{array}{c}\text { Coef. } \\
\text { Asim. }\end{array}$ & Curtosis & Jarque-Bera & P-value \\
\hline Alfa & 0.0001 & 0.0484 & -35.9928 & 1718.8850 & 371000000.00 & 0.000 \\
\hline Bimbo & 0.0002 & 0.0315 & -27.7152 & 1217.7010 & 186000000.00 & 0.000 \\
\hline Cemex & -0.0004 & 0.0325 & -6.3221 & 141.9362 & 2449923.00 & 0.000 \\
\hline Comercial Mexicana & 0.0004 & 0.0376 & -15.7781 & 575.7829 & 41422441.00 & 0.000 \\
\hline Consorcio Ara & -0.0004 & 0.0334 & -22.2205 & 901.7301 & 102000000.00 & 0.000 \\
\hline Corporación Geo & 0.0000 & 0.0400 & -21.3789 & 855.7748 & 91769549.00 & 0.000 \\
\hline Empresas Ica & 0.0008 & 0.0446 & 22.2847 & 902.1950 & 102000000.00 & 0.000 \\
\hline Fomento EconMex & 0.0004 & 0.0274 & -21.0478 & 838.0277 & 87992073.00 & 0.000 \\
\hline G Financiero Banorte & 0.0006 & 0.0357 & -19.1167 & 746.2015 & 69710861.00 & 0.000 \\
\hline G Financiero Inbursa & -0.0001 & 0.0316 & -16.8135 & 527.4426 & 34762998.00 & 0.000 \\
\hline Grupo Carso & 0.0002 & 0.0332 & -16.5693 & 506.0711 & 31994741.00 & 0.000 \\
\hline Grupo Elektra & 0.0014 & 0.0388 & 24.1982 & 1018.6860 & 130000000.00 & 0.000 \\
\hline Grupo México & 0.0000 & 0.0393 & -14.1791 & 395.0286 & 19446509.00 & 0.000 \\
\hline Grupo Modelo & 0.0005 & 0.0178 & 0.2205 & 9.9764 & 6150.81 & 0.000 \\
\hline Grupo Televisa & 0.0003 & 0.0203 & 0.0803 & 8.4038 & 3678.93 & 0.000 \\
\hline Kimberly Clark México & 0.0001 & 0.0256 & -24.1702 & 1008.8310 & 128000000.00 & 0.000 \\
\hline Organización Soriana & 0.0001 & 0.0280 & -18.7464 & 718.2651 & 64575113.00 & 0.000 \\
\hline Telmex & -0.0003 & 0.0239 & -14.4872 & 372.0754 & 17251947.00 & 0.000 \\
\hline TV Azteca & 0.0001 & 0.0215 & -0.5897 & 12.9664 & 12678.04 & 0.000 \\
\hline Wal Mart de México & 0.0002 & 0.0261 & -12.5311 & 335.6286 & 14006097.00 & 0.000 \\
\hline & 0.0006 & 0.0138 & 0.0147 & 7.8051 & 2906.42 & 0.000 \\
\hline
\end{tabular}

El cuadro 2 muestra la estadística descriptiva de las series considerando el periodo del 3 de octubre de 2000 al 09 de agosto de 2007. Esta cuadro es importante porque provee escasa evidencia en favor de la EMH. Esto ocurre porque las medias de los rendimientos a nivel agregado y desagregado, aunque tendientes a cero, son mayores que las registradas en el cuadro 1. Además, las desviaciones estándar tienden a ser consistentemente menores (lo cual sugiere la existencia de clusters de volatilidad). El cuadro también muestra que 17 series exhiben un sesgo negativo y, las restantes, un sesgo positivo. Asimismo, el cuadro confirma que las series son leptocúrticas y que ninguna se distribuye normalmente. 


\section{Cuadro 2}

\section{Estadística descriptiva de los rendimientos de los activos para el periodo del 2 de octubre de 2000 al 9 de agosto de 2007 y prueba de normalidad de Jarque-Bera}

\begin{tabular}{|l|r|r|r|r|r|r|}
\hline \multicolumn{1}{|c|}{ Activo Bursátil } & Media & $\begin{array}{c}\text { Desv. } \\
\text { Est. }\end{array}$ & $\begin{array}{c}\text { Coef. } \\
\text { Asim. }\end{array}$ & Curtosis & Jarque-Bera & P-value \\
\hline Alfa & 0.0008 & 0.0233 & -0.5777 & 11.2462 & 4966.09 & 0.000 \\
\hline Bimbo & 0.0008 & 0.0185 & 0.2769 & 7.2067 & 1289.44 & 0.000 \\
\hline Cemex & 0.0000 & 0.0289 & -15.8433 & 380.7933 & 10294792.00 & 0.000 \\
\hline Comercial Mexicana & 0.0006 & 0.0206 & 0.1735 & 6.3750 & 824.49 & 0.000 \\
\hline Consorcio Ara & 0.0000 & 0.0379 & -26.5138 & 947.6066 & 64111081.00 & 0.000 \\
\hline Corporación Geo & 0.0008 & 0.0458 & -25.1411 & 881.6503 & 55477474.00 & 0.000 \\
\hline Empresas Ica & 0.0020 & 0.0536 & 22.4886 & 755.2449 & 40675506.00 & 0.000 \\
\hline Fomento EconMex & 0.0000 & 0.0316 & -24.0933 & 832.0268 & 49393127.00 & 0.000 \\
\hline G Financiero Banorte & 0.0008 & 0.0393 & -25.3090 & 893.2874 & 56954332.00 & 0.000 \\
\hline G Financiero Inbursa & -0.0002 & 0.0325 & -20.8989 & 681.2485 & 33074136.00 & 0.000 \\
\hline Grupo Carso & 0.0002 & 0.0324 & -22.0722 & 741.2443 & 39175536.00 & 0.000 \\
\hline Grupo Elektra & 0.0018 & 0.0456 & 26.2820 & 941.7286 & 63314670.00 & 0.000 \\
\hline Grupo México & 0.0004 & 0.0362 & -15.4013 & 460.8551 & 15082802.00 & 0.000 \\
\hline Grupo Modelo & 0.0006 & 0.0162 & -0.1207 & 8.0573 & 1836.08 & 0.000 \\
\hline Grupo Televisa & 0.0004 & 0.0209 & -0.2707 & 8.2519 & 1996.57 & 0.000 \\
\hline Kimberly Clark México & 0.0003 & 0.0153 & -1.0020 & 16.0024 & 12396.73 & 0.000 \\
\hline Organización Soriana & 0.0001 & 0.0319 & -22.2406 & 749.5003 & 40055655.00 & 0.000 \\
\hline Telmex & -0.0002 & 0.0254 & -16.1884 & 409.6314 & 11918212.00 & 0.000 \\
\hline TralMart de México & 0.0000 & 0.0239 & -0.9312 & 12.8291 & 7168.16 & 0.000 \\
\hline & 0.0004 & 0.0254 & -12.9346 & 366.4204 & 9507758.00 & 0.000 \\
\hline & 0.0009 & 0.0126 & -0.1908 & 5.4869 & 453.41 & 0.000 \\
\hline
\end{tabular}

El cuadro 3 muestra la estadística descriptiva de las series de rendimientos bursátiles considerando el periodo del 10 de agosto de 2007 al 10 de octubre de 2012. En este cuadro, las medias de los rendimientos tienden a ser menores que las registradas en el cuadro 1. Las desviaciones estándar, por su parte, tienden a ser mayores. Por tanto, las estimaciones de los tres cuadros sugieren la existencia de movimientos conjuntos de las medias y las varianzas. El cuadro también muestra que catorce series exhiben un sesgo negativo y las restantes un sesgo positivo. Nuevamente, el cuadro confirma que todas las series son leptocúrticas y que ninguna se distribuye normalmente. 


\section{Cuadro 3}

\section{Estadística descriptiva de los rendimientos de los activos para el periodo del 10 de agosto de 2007 al 10 de octubre de 2012 y prueba de normalidad de Jarque-Bera}

\begin{tabular}{|l|r|r|r|r|r|r|}
\hline \multicolumn{1}{|c|}{ Activo Bursátil } & Media & $\begin{array}{c}\text { Desv. } \\
\text { Est. }\end{array}$ & $\begin{array}{c}\text { Coef. } \\
\text { Asim. }\end{array}$ & Curtosis & Jarque-Bera & P-value \\
\hline Alfa & -0.0009 & 0.0687 & -29.1413 & 980.6914 & 52000823.00 & 0.000 \\
\hline Bimbo & -0.0005 & 0.0431 & -25.2192 & 809.3410 & 35383399.00 & 0.000 \\
\hline Cemex & -0.0009 & 0.0367 & 0.0632 & 9.0822 & 2006.23 & 0.000 \\
\hline Comercial Mexicana & 0.0001 & 0.0522 & -13.7080 & 359.9467 & 6947480.00 & 0.000 \\
\hline Consorcio Ara & -0.0011 & 0.0262 & -0.4349 & 21.1107 & 17821.12 & 0.000 \\
\hline Corporación Geo & -0.0010 & 0.0309 & -0.2070 & 7.7812 & 1248.48 & 0.000 \\
\hline Empresas Ica & -0.0007 & 0.0285 & -0.0687 & 21.3620 & 18278.00 & 0.000 \\
\hline Fomento EconMex & 0.0009 & 0.0206 & -0.2092 & 9.7216 & 2458.63 & 0.000 \\
\hline G Financiero Banorte & 0.0004 & 0.0304 & 0.1122 & 14.5548 & 7240.20 & 0.000 \\
\hline G Financiero Inbursa & 0.0001 & 0.0303 & -10.0752 & 251.3793 & 3366247.00 & 0.000 \\
\hline Grupo Carso & 0.0000 & 0.0344 & -10.4641 & 258.7697 & 3569951.00 & 0.000 \\
\hline Grupo Elektra & 0.0007 & 0.0275 & 0.0602 & 10.3852 & 2957.38 & 0.000 \\
\hline Grupo México & -0.0003 & 0.0432 & -12.9432 & 332.4906 & 5921403.00 & 0.000 \\
\hline Grupo Modelo & 0.0005 & 0.0197 & 0.4656 & 10.5469 & 3134.51 & 0.000 \\
\hline Grupo Televisa & 0.0001 & 0.0194 & 0.6577 & 8.6072 & 1798.15 & 0.000 \\
\hline Kimberly Clark México & -0.0003 & 0.0349 & -22.1153 & 680.5870 & 24994407.00 & 0.000 \\
\hline Organización Soriana & 0.0001 & 0.0218 & -0.2949 & 7.4041 & 1070.31 & 0.000 \\
\hline Telmex & -0.0005 & 0.0218 & -10.6274 & 256.1973 & 3499725.00 & 0.000 \\
\hline TV Azteca & 0.0002 & 0.0181 & 0.5286 & 9.3041 & 2214.95 & 0.000 \\
\hline IPC Mart de México & 0.0000 & 0.0270 & -12.0631 & 301.6851 & 4867629.00 & 0.000 \\
\hline & 0.0003 & 0.0151 & 0.1924 & 8.9055 & 1898.57 & 0.000 \\
\hline
\end{tabular}

Finalmente debe señalarse que el análisis de estadística descriptiva sugiere que hay escasa evidencia para sustentar la hipótesis de eficiencia en el mercado bursátil mexicano. Las medias de los rendimientos, si bien tienden a cero, son positivas durante los periodos considerados. Asimismo, la evidencia muestra que las series tienden a manifestar curtosis excesivas, clusters de volatilidad, asimetrías, distribuciones no normales, volatilidades no constantes y movimientos conjuntos de medias y varianzas. Estas características implican que las series de rendimientos pudieran ser analizables mediante modelos de la familia ARCH (y, por tanto, potencialmente inconsistentes con la EMH). 


\section{Análisis de estacionariedad}

Metodológicamente, el análisis de estacionariedad se usa para analizar la pertinencia de modelar las series bursátiles y la validez de la hipótesis de eficiencia (EMH). Como es conocido, la modelación econométrica de series requiere que las mismas sean integradas de orden cero I(0). Asimismo, la EMH requiere que las series de precios bursátiles no sean estacionarias. En este contexto, aquí se usa el test Aumentado de Dickey-Fuller (ADF) para analizar el orden de integración de las series de logaritmos de precios. Las estimaciones del test ADF, considerando las series completas, se muestran en el cuadro 4. Las estimaciones obtenidas considerando las series en los otros periodos se muestran en el cuadro 5.

\section{Cuadro 4}

Prueba ADF para las series de los logaritmos de precios (niveles) y de los rendimientos (diferencias) para el periodo del 2 de octubre de 2000 al 10 de octubre de 2012

\begin{tabular}{|c|c|c|c|c|c|}
\hline \multirow{3}{*}{ Activo Bursátil } & \multicolumn{5}{|c|}{$02 / 10 / 2000-09 / 08 / 2007$} \\
\hline & \multicolumn{2}{|c|}{ Niveles } & \multicolumn{2}{|c|}{ Diferencias } & \multirow{2}{*}{$\mathbf{I}(\mathbf{d})$} \\
\hline & Prob & Rezagos & Prob & Rezagos & \\
\hline Alfa & 0.5884 & 0 & 0.0001 & 0 & 1 \\
\hline Bimbo & 0.7162 & 0 & 0.0001 & 0 & 1 \\
\hline Cemex & 0.2891 & 1 & 0.0001 & 0 & 1 \\
\hline Comercial Mexicana & 0.7196 & 4 & 0.0000 & 3 & 1 \\
\hline Consorcio Ara & 0.3423 & 0 & 0.0001 & 0 & 1 \\
\hline Corporación Geo & 0.2145 & 1 & 0.0001 & 0 & 1 \\
\hline Empresas Ica & 0.7710 & 0 & 0.0001 & 0 & 1 \\
\hline Fomento Econ Mex & 0.8524 & 0 & 0.0001 & 0 & 1 \\
\hline G Financiero Banorte & 0.1326 & 0 & 0.0001 & 0 & 1 \\
\hline G Financiero Inbursa & 0.1424 & 0 & 0.0001 & 0 & 1 \\
\hline Grupo Carso & 0.0197 & 0 & 0.0001 & 0 & 0 \\
\hline Grupo Elektra & 0.9448 & 1 & 0.0001 & 0 & 1 \\
\hline Grupo México & 0.5982 & 0 & 0.0001 & 0 & 1 \\
\hline Grupo Modelo & 0.2171 & 2 & 0.0001 & 1 & 1 \\
\hline Grupo Televisa & 0.2718 & 2 & 0.0000 & 1 & 1 \\
\hline Kimberly Clark México & 0.2796 & 0 & 0.0001 & 0 & 1 \\
\hline Organización Soriana & 0.1100 & 0 & 0.0001 & 0 & 1 \\
\hline Telmex & 0.0441 & 0 & 0.0001 & 0 & 0 \\
\hline
\end{tabular}




\begin{tabular}{|c|c|c|c|c|c|}
\hline TV Azteca & 0.0691 & 0 & 0.0001 & 0 & 1 \\
\hline Wal Mart de México & 0.0160 & 0 & 0.0001 & & 0 \\
\hline IPC & 0.9946 & 1 & 0.0001 & 0 & 1 \\
\hline
\end{tabular}

Nota: La hipótesis nula asociada a la prueba ADF se rechaza cuando Prob $<0.05$.

Los cuadros 4 y 5 muestran que la gran mayoría de las series de logaritmos de precios son I(1). Esto conlleva a que la mayoría de los activos aparentemente puedan validar el cumplimiento de la EMH. Particularmente, el cuadro 4 muestra que, si se considera las series completas, hay 18 series de precios I(1). El cuadro 5 muestra que durante el periodo de "calma", entre el 2 de octubre de 2000 y el 9 de agosto de 2007, hay 19 series I(1). Asimismo muestra que, si se considera el periodo de "inestabilidad", entre el 10 de agosto de 2007 y el 10 de octubre de 2012 hay 18 series I(1). Por tanto, la comparación muestra que el número de series que validan la EMH ha tendido a disminuir a lo largo del tiempo.

\section{Cuadro 5}

Prueba ADF para las series de los logaritmos de precios (niveles) y de los rendimientos (diferencias) para los periodos del 2 de octubre de 2000 al 9 de agosto de 2007, y del 10 de agosto de 2007 al 10 de octubre de 2012

\begin{tabular}{|c|c|c|c|c|c|}
\hline \multirow{3}{*}{ Activo Bursátil } & \multicolumn{5}{|c|}{$02 / 10 / 2000-09 / 08 / 2007$} \\
\hline & \multicolumn{2}{|c|}{ Niveles } & \multicolumn{2}{|c|}{ Diferencias } & \multirow{2}{*}{$\mathrm{I}(\mathrm{d})$} \\
\hline & Prob & Rezagos & Prob & Rezagos & \\
\hline Alfa & 0.1802 & 2 & 0.0000 & 0 & 1 \\
\hline Bimbo & 0.4217 & 0 & 0.0000 & 0 & 1 \\
\hline Cemex & 0.2047 & 0 & 0.0000 & 0 & 1 \\
\hline Comercial Mexicana & 0.1450 & 0 & 0.0000 & 0 & 1 \\
\hline Consorcio Ara & 0.6153 & 0 & 0.0000 & 0 & 1 \\
\hline Corporación Geo & 0.4042 & 0 & 0.0000 & 0 & 1 \\
\hline Empresas Ica & 0.9573 & 0 & 0.0000 & 0 & 1 \\
\hline Fomento Econ Mex & 0.6321 & 0 & 0.0000 & 0 & 1 \\
\hline G Financiero Banorte & 0.2841 & 0 & 0.0000 & 0 & 1 \\
\hline G Financiero Inbursa & 0.4746 & 0 & 0.0000 & 0 & 1 \\
\hline Grupo Carso & 0.2125 & 0 & 0.0000 & 0 & 1 \\
\hline Grupo Elektra & 0.2355 & 1 & 0.0000 & 0 & 1 \\
\hline Grupo México & 0.7669 & 0 & 0.0000 & 0 & 1 \\
\hline
\end{tabular}


Economía Informa núm. 390 enero - febrero • 2015

\begin{tabular}{|c|c|c|c|c|c|}
\hline Grupo Modelo & 0.2977 & 0 & 0.0000 & 1 & 1 \\
\hline Grupo Televisa & 0.0963 & 1 & 0.0000 & 0 & 1 \\
\hline Kimberly Clark México & 0.0483 & 0 & 0.0000 & 0 & 0 \\
\hline Organización Soriana & 0.0909 & 0 & 0.0000 & 0 & 1 \\
\hline Telmex & 0.0452 & 0 & 0.0000 & 0 & 0 \\
\hline TV Azteca & 0.0681 & 0 & 0.0000 & 0 & 1 \\
\hline Wal Mart de México & 0.0599 & 0 & 0.0000 & 0 & 1 \\
\hline IPC & 0.3943 & 1 & 0.0000 & 0 & 1 \\
\hline \multirow{3}{*}{ Activo Bursátil } & \multicolumn{5}{|c|}{$10 / 08 / 2007-10 / 10 / 2012$} \\
\hline & \multicolumn{2}{|c|}{ Niveles } & \multicolumn{2}{|c|}{ Diferencias } & \multirow{2}{*}{ I(d) } \\
\hline & Prob & Rezagos & Prob & Rezagos & \\
\hline Alfa & 0.4209 & 0 & 0.0000 & 0 & 1 \\
\hline Bimbo & 0.4691 & 0 & 0.0000 & 0 & 1 \\
\hline Cemex & 0.1877 & 1 & 0.0000 & 0 & 1 \\
\hline Comercial Mexicana & 0.5978 & 4 & 0.0000 & 3 & 1 \\
\hline Consorcio Ara & 0.0587 & 0 & 0.0000 & 0 & 1 \\
\hline Corporación Geo & 0.2065 & 0 & 0.0000 & 0 & 1 \\
\hline Empresas Ica & 0.2570 & 1 & 0.0000 & 0 & 1 \\
\hline Fomento Econ Mex & 0.1020 & 0 & 0.0000 & 0 & 1 \\
\hline G Financiero Banorte & 0.7790 & 0 & 0.0000 & 0 & 1 \\
\hline G Financiero Inbursa & 0.2031 & 0 & 0.0000 & 0 & 1 \\
\hline Grupo Carso & 0.0237 & 0 & 0.0000 & 0 & 0 \\
\hline Grupo Elektra & 0.1942 & 1 & 0.0000 & 0 & 1 \\
\hline Grupo México & 0.4806 & 0 & 0.0000 & 0 & 1 \\
\hline Grupo Modelo & 0.2699 & 2 & 0.0000 & 1 & 1 \\
\hline Grupo Televisa & 0.0353 & 0 & 0.0000 & 0 & 0 \\
\hline Kimberly Clark México & 0.5341 & 0 & 0.0000 & 0 & 1 \\
\hline Organización Soriana & 0.0490 & 1 & 0.0000 & 0 & 0 \\
\hline Telmex & 0.2330 & 0 & 0.0000 & 0 & 1 \\
\hline TV Azteca & 0.1674 & 0 & 0.0000 & 0 & 1 \\
\hline Wal Mart de México & 0.0836 & 0 & 0.0000 & 0 & 1 \\
\hline IPC & 0.5364 & 0 & 0.0000 & 0 & 1 \\
\hline
\end{tabular}

Nota: La hipótesis nula asociada a la prueba ADF se rechaza cuando Prob $<0.05$.

Las series de logaritmos de precios que son $\mathrm{I}(0)$ son susceptibles de ser descritas y analizadas de manera directa mediante modelos econométricos. Por tanto refieren a activos individuales que no cumplen con la hipótesis de eficiencia. 
Si se considera la totalidad del periodo analizado, estas series corresponden a Grupo Carso, Telmex y Walmart de México. Si se considera el periodo de "calma", las series corresponden a Kimberly Clark México y Telmex. Si se considera el periodo de "inestabilidad", las series corresponden a Grupo Carso, Organización Soriana y Grupo Televisa. Por tanto, el número de series a nivel desagregado que no validan la EMH es pequeño, pero creciente, en el tiempo.

Analíticamente, los resultados estimados tienen implicaciones sobre el mercado bursátil mexicano. Particularmente la validación de que la mayoría de las series de logaritmos de precios son I(1) sugiere que la hipótesis de eficiencia prevalece en el mercado a nivel agregado y desagregado. Sin embargo, los resultados también sugieren que la eficiencia del mismo ha tendido a disminuir a nivel desagregado desde agosto de 2007. Econométricamente, la validación de que las series de precios sean I(1) conlleva a que las diferencias de las mismas sean modelables. Esto significa que la mayoría de las series de rendimientos bursátiles son susceptibles de ser descritas y analizadas estadísticamente.

\section{Ejercicios de modelación y análisis econométricos}

Los modelos GARCH multivariados (MGARCH) usados en esta investigación comparten una estructura similar para efectos de estimación econométrica. En todos los modelos, los vectores de la media condicional requieren estimar los coeficientes $\phi_{i 1}$ y $\rho_{i}$. Asimismo, requieren hacer estimaciones de las matrices de varianza-covarianza. Por comparabilidad y simplicidad, la dinámica conjunta de las series de rendimientos se describe usando los doce modelos multivariados señalados anteriormente. Al igual que en los análisis anteriores, las estimaciones se agrupan por periodos. Estas estimaciones están sintetizadas en los cuadros 67 y 8. La comparación econométrica de las estimaciones se realiza con base en el cuadro 9.

Los cuadros 6, 7 y 8 muestran las estimaciones obtenidas mediante los modelos multivariados para las series de rendimientos a nivel agregado y desagregado. El cuadro 6 incluye las estimaciones considerando la totalidad del periodo analizado. El cuadro 7 incluye las estimaciones considerando el periodo de "calma" financiera. El cuadro 8 incluye las estimaciones considerando el periodo de "inestabilidad". Los activos incluidos en las estimaciones fueron aquellos cuyas series de precios fueran I(1) y cuyas series de 
rendimientos hubieran manifestado efectos ARCH. ${ }^{15}$ Así, el número de activos incluidos en las estimaciones de los cuadros 6,7 y 8 son, respectivamente, 5, 7 y $11 .^{16}$

El cuadro 6 muestra las estimaciones que describen la dinámica del conjunto de series de rendimientos bursátiles considerando el periodo completo. Este cuadro provee valores de referencia para evaluar la hipótesis de eficiencia y la distribución de las perturbaciones. El cuadro muestra que los rendimientos tienden a estar correlacionados entre sí: Todas las estimaciones de $\rho_{i}$ y la mayoría de $\phi_{i 1}$ son significativas. Por tanto, la evidencia provee escasa evidencia para validar la hipótesis de eficiencia. Además, el cuadro provee evidencia de que las perturbaciones se distribuyen siguiendo una distribución $t$ de Student multivariada: Todas las estimaciones referidas a los grados de libertad son significativas.

\section{Cuadro 6}

Estimaciones obtenidas mediante los modelos GARCH multivariados para las series de rendimientos durante el periodo del 2 de octubre de 2000 al 10 de octubre de 2012

\begin{tabular}{|c|c|c|c|c|c|c|c|c|c|c|}
\hline & & & $\begin{array}{r}\text { AR (1) } \\
\text { GARCH( }\end{array}$ & & $\begin{array}{r}\text { AR }(1 \\
\text { TGARCH }\end{array}$ & $(1,1)$ & $\begin{array}{r}\text { AR }(1 \\
\text { GARCH( } \\
\text { Stude }\end{array}$ & $\begin{array}{l}- \\
\text { 1)-t } \\
n t\end{array}$ & $\begin{array}{r}\operatorname{AR}(1) \\
\text { TGARCH( } \\
\text { Stude }\end{array}$ & \\
\hline & & $\rho$ & 0.013995 & & 0.0204 & $* *$ & 0.0118 & & 0.0142 & $*$ \\
\hline & DVECH & P. Estimados & 6 & & 6 & & 6 & & 6 & \\
\hline & & P. Significativos & 5 & & 2 & & 5 & & 6 & \\
\hline & & $\rho$ & 0.0087 & & 0.0131 & $*$ & 0.0131 & $*$ & 0.0138 & $*$ \\
\hline$\sum^{0}$ & $\mathrm{CCC}$ & P. Estimados & 6 & & 6 & & 6 & & 6 & \\
\hline & & P. Significativos & 5 & & 2 & & 6 & & 2 & \\
\hline & & $\rho$ & 0.0223 & $* * *$ & 0.0245 & $* * *$ & 0.0098 & $* * *$ & 0.0120 & \\
\hline & DBEKK & P. Estimados & 6 & & 6 & & 6 & & 6 & \\
\hline & & P. Significativos & 5 & & 6 & & 4 & & 4 & \\
\hline
\end{tabular}

${ }^{15}$ Las series de rendimientos que muestran efectos ARCH fueron determinadas con base en la prueba del Multiplicador de Lagrange (LM). La hipótesis nula asociada a esta prueba es que no hay efectos ARCH.

${ }^{16}$ Los cinco activos incluidos en el cuadro 6 incluyen a Comercial Mexicana, Grupo Modelo, Grupo Televisa, TV Azteca y el IPC. Los siete activos incluidos en el cuadro 7 incluyen a Alfa, Bimbo, Comercial Mexicana, Grupo Modelo, Grupo Televisa, TV Azteca y el IPC. Los once activos incluidos en el cuadro 8 incluyen a Consorcio Ara, Cemex, Comercial Mexicana, Grupo Elektra, Fomento Econ. Mex., Corporación Geo, G Financiero Banorte, Grupo Modelo, Empresas Ica, TV Azteca y el IPC. Adviértase que el número de series modelables, i.e. ineficientes, se incrementó desde 2007. 


\begin{tabular}{|c|c|c|c|c|c|c|}
\hline \multirow{6}{*}{ 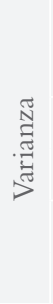 } & \multirow{2}{*}{ DVECH } & P. Estimados & 45 & 60 & 45 & 46 \\
\hline & & P. Significativos & 45 & 56 & 45 & 43 \\
\hline & \multirow{2}{*}{$\mathrm{CCC}$} & P. Estimados & 25 & 30 & 25 & 30 \\
\hline & & P. Significativos & 25 & 29 & 25 & 28 \\
\hline & \multirow{2}{*}{ DBEKK } & P. Estimados & 11 & 16 & 11 & 16 \\
\hline & & P. Significativos & 11 & 16 & 11 & 15 \\
\hline \multirow{6}{*}{ 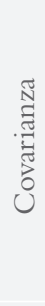 } & \multirow{2}{*}{ DVECH } & P. Estimados & 45 & 60 & 45 & 46 \\
\hline & & P. Significativos & 45 & 56 & 45 & 43 \\
\hline & \multirow{2}{*}{ CCC } & P. Estimados & 25 & 30 & 25 & 30 \\
\hline & & P. Significativos & 25 & 29 & 25 & 28 \\
\hline & \multirow{2}{*}{ DBEKK } & P. Estimados & 11 & 16 & 11 & 16 \\
\hline & & P. Significativos & 11 & 16 & 11 & 15 \\
\hline \multirow{6}{*}{ 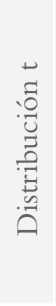 } & \multirow{2}{*}{ DVECH } & P. Estimados & & & 1 & 1 \\
\hline & & P. Significativos & & & 1 & 1 \\
\hline & \multirow{2}{*}{ CCC } & P. Estimados & & & 1 & 1 \\
\hline & & P. Significativos & & & 1 & 1 \\
\hline & \multirow[b]{2}{*}{ DBEKK } & P. Estimados & & & 1 & 1 \\
\hline & & P. Significativos & & & 1 & 1 \\
\hline
\end{tabular}

Notas: ${ }^{* *}$ y $* * *$ denotan niveles de significancia del 10, 5 y 1 por ciento respectivamente. Las estimaciones se refieren a los 5 activos cuyas series de rendimientos manifestaron efectos ARCH.

El cuadro 7 muestra las estimaciones que describen la dinámica del conjunto de series de rendimientos bursátiles considerando el periodo del 3 de octubre de 2000 al 9 de agosto de 2007. Este cuadro confirma los resultados obtenidos anteriormente. Los rendimientos tienden a estar correlacionados en el tiempo ya que todas las estimaciones de $\rho_{i}$ y la mayoría de $\phi_{i 1}$ son significativas. Por tanto, la evidencia confirma que existe escasa evidencia para validar la hipótesis de eficiencia. Además, el cuadro confirma que las perturbaciones se distribuyen siguiendo una distribución $t$ de Student multivariada. Al igual que antes, todas las estimaciones referidas a los grados de libertad son significativas. 


\section{Cuadro 7}

Estimaciones obtenidas mediante los modelos GARCH multivariados para las series de rendimientos durante el periodo del 2 de octubre de 2000 al 9 de agosto de 2007

\begin{tabular}{|c|c|c|c|c|c|c|c|c|c|c|}
\hline & & & $\begin{array}{r}\text { AR }( \\
\text { GARCH }\end{array}$ & & $\begin{array}{r}\text { AR }(1 \\
\text { TGARCP }\end{array}$ & - & $\begin{array}{r}\text { AR }( \\
\text { GARCH } \\
\text { Stud }\end{array}$ & $\begin{array}{l}- \\
\text {,1)-t } \\
n t\end{array}$ & $\begin{array}{r}\text { AR }(1 \\
\text { TGARCH } \\
\text { Stud }\end{array}$ & \\
\hline & & $\rho$ & 0.0275 & $* * *$ & 0.0350 & $* * *$ & 0.0189 & $* *$ & 0.0217 & $* * *$ \\
\hline & DVECH & P. Estimados & 8 & & 8 & & 8 & & 8 & \\
\hline & & P. Significativos & 7 & & 7 & & 4 & & 7 & \\
\hline & & $\rho$ & 0.0291 & $* * *$ & 0.0323 & $* * *$ & 0.0226 & $* * *$ & 0.0237 & $* * *$ \\
\hline$\stackrel{\overrightarrow{0}}{\pi}$ & $\mathrm{CCC}$ & P. Estimados & 8 & & 8 & & 8 & & 8 & \\
\hline & & P. Significativos & 7 & & 4 & & 5 & & 3 & \\
\hline & & $\rho$ & 0.0317 & $* * *$ & 0.0308 & $* * *$ & 0.0197 & $* *$ & 0.0195 & $* *$ \\
\hline & DBEKK & P. Estimados & 8 & & 8 & & 8 & & 8 & \\
\hline & & P. Significativos & 6 & & 6 & & 4 & & 4 & \\
\hline & & P. Estimados & 84 & & 43 & & 84 & & 43 & \\
\hline & DVECH & P. Significativos & 78 & & 26 & & 75 & & 19 & \\
\hline$\stackrel{N}{\Xi}$ & & P. Estimados & 42 & & 49 & & 42 & & 49 & \\
\hline$\frac{1}{>}$ & . & P. Significativos & 42 & & 46 & & 42 & & 45 & \\
\hline & & P. Estimados & 15 & & 22 & & 15 & & 22 & \\
\hline & DDLКК & P. Significativos & 15 & & 22 & & 15 & & 17 & \\
\hline & & P. Estimados & 84 & & 85 & & 84 & & 85 & \\
\hline & DVECH & P. Significativos & 78 & & 68 & & 75 & & 61 & \\
\hline.$\widetilde{\Xi ี ~}$ & & P. Estimados & 42 & & 49 & & 42 & & 49 & \\
\hline है & . & P. Significativos & 42 & & 46 & & 42 & & 45 & \\
\hline & & P. Estimados & 15 & & 22 & & 15 & & 22 & \\
\hline & ВБеки & P. Significativos & 15 & & 22 & & 15 & & 17 & \\
\hline & & P. Estimados & & & & & 1 & & 1 & \\
\hline 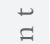 & DVECT & P. Significativos & & & & & 1 & & 1 & \\
\hline$\stackrel{\vec{J}}{g}$ & & P. Estimados & & & & & 1 & & 1 & \\
\hline $\bar{E}$ & . & P. Significativos & & & & & 1 & & 1 & \\
\hline$\ddot{a}$ & & P. Estimados & & & & & 1 & & 1 & \\
\hline & УDLКК & P. Significativos & & & & & 1 & & 1 & \\
\hline
\end{tabular}

Notas: *** y *** denotan niveles de significancia del 10, 5 y 1 por ciento respectivamente. Las estimaciones se refieren a los 7 activos cuyas series de rendimientos manifestaron efectos ARCH. 
El cuadro 8 muestra las estimaciones que describen la dinámica del conjunto de series de rendimientos bursátiles considerando el periodo del 10 de agosto de 2007 al 10 de octubre de 2012. Este cuadro también confirma los resultados obtenidos anteriormente. Al igual que antes, los rendimientos tienden a estar significativamente correlacionados entre sí. Sin embargo, debe mencionarse que las magnitudes absolutas de $\rho_{i}$ obtenidas tienden a ser mayores que las obtenidas en los periodos previos. Además, el cuadro también confirma que las perturbaciones se distribuyen siguiendo una distribución $t$ de Student multivariada. Asimismo, todas las estimaciones referidas a los grados de libertad son significativas.

\section{Cuadro 8}

Estimaciones obtenidas mediante los modelos garch

multivariados para las series de rendimientos durante el periodo del 10 de agosto de 2007 al 10 de octubre de 2012

\begin{tabular}{|c|c|c|c|c|c|c|c|c|c|c|}
\hline & & & $\begin{array}{r}\text { AR }(1 \\
\text { GARCH }\end{array}$ & & $\begin{array}{r}\operatorname{AR}(1) \\
\text { TGARCH }\end{array}$ & & $\begin{array}{r}\text { AR }( \\
\text { GARCH } \\
\text { Stud }\end{array}$ & $\begin{array}{l}- \\
\text {,1)-t } \\
n t\end{array}$ & $\begin{array}{r}\operatorname{AR}(1 \\
\text { TGARCH } \\
\text { Stud }\end{array}$ & 1)-t \\
\hline \multirow{9}{*}{ 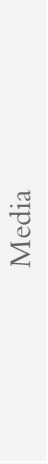 } & \multirow{3}{*}{ DVECH } & $\rho$ & 0.0453 & $* * *$ & -0.0069 & $* *$ & 0.0296 & $* *$ & 0.0395 & $* * *$ \\
\hline & & P. Estimados & 12 & & 12 & & 12 & & 12 & \\
\hline & & P. Significativos & 4 & & 8 & & 4 & & 10 & \\
\hline & \multirow{3}{*}{$\mathrm{CCC}$} & $\rho$ & 0.0463 & $* * *$ & 0.0459 & $* * *$ & 0.0370 & $* * *$ & 0.0368 & $* * *$ \\
\hline & & P. Estimados & 12 & & 12 & & 12 & & 12 & \\
\hline & & P. Significativos & 7 & & 4 & & 4 & & 4 & \\
\hline & \multirow{3}{*}{ DBEKK } & $\rho$ & 0.0474 & $* * *$ & 0.0525 & $* * *$ & 0.0397 & $* * *$ & 0.0358 & $* * *$ \\
\hline & & P. Estimados & 12 & & 12 & & 12 & & 12 & \\
\hline & & P. Significativos & 7 & & 6 & & 5 & & 4 & \\
\hline \multirow{6}{*}{ 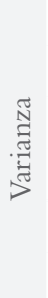 } & \multirow{2}{*}{ DVECH } & P. Estimados & 198 & & 89 & & 198 & & 89 & \\
\hline & & P. Significativos & 189 & & 70 & & 191 & & 79 & \\
\hline & \multirow{2}{*}{$\mathrm{CCC}$} & P. Estimados & 88 & & 99 & & 88 & & 99 & \\
\hline & & P. Significativos & 88 & & 98 & & 88 & & 95 & \\
\hline & \multirow{2}{*}{ DBEKK } & P. Estimados & 23 & & 34 & & 23 & & 34 & \\
\hline & & P. Significativos & 23 & & 34 & & 23 & & 33 & \\
\hline
\end{tabular}


Economía Informa núm. 390 enero - febrero • 2015

\begin{tabular}{|c|c|c|c|c|c|}
\hline \multirow{2}{*}{ DVECH } & P. Estimados & 198 & 199 & 198 & 199 \\
\hline & P. Significativos & 189 & 180 & 191 & 172 \\
\hline \multirow{2}{*}{$\mathrm{CCC}$} & P. Estimados & 88 & 99 & 88 & 99 \\
\hline & P. Significativos & 88 & 98 & 88 & 95 \\
\hline \multirow{2}{*}{ DBEKK } & P. Estimados & 23 & 34 & 23 & 34 \\
\hline & P. Significativos & 23 & 34 & 23 & 33 \\
\hline \multirow[b]{2}{*}{ DVECH } & P. Estimados & & & 1 & 1 \\
\hline & P. Significativos & & & 1 & 1 \\
\hline \multirow{2}{*}{ CCC } & P. Estimados & & & 1 & 1 \\
\hline & P. Significativos & & & 1 & 1 \\
\hline \multirow{2}{*}{ DBEKK } & P. Estimados & & & 1 & 1 \\
\hline & P. Significativos & & & 1 & 1 \\
\hline
\end{tabular}

El cuadro 9 muestra y compara los estimadores de bondad de ajuste para cada una de las estimaciones realizadas. Esta evaluación se hace comparando los valores absolutos de los estimadores. La mayoría de las comparaciones muestran que los modelos de tipo CCC (Constant Conditional Correlation) son los que tienden a describir mejor la dinámica conjunta de las series. Particularmente, las comparaciones entre modelos similares muestran que la especificación TGARCH parece caracterizar de mejor manera a la volatilidad de las series. Más aún, las comparaciones confirman que la distribución de densidad $t$ de Student multivariada describe de mejor manera a las perturbaciones.

La consistencia de los resultados econométricos sugiere que hay patrones definidos de comportamiento de las series de rendimientos. Particularmente, el número creciente de series modelables confirma que la ineficiencia ha tendido a aumentar desde 2007. La predominancia de los modelos de tipo CCC sugiere que las correlaciones condicionales explican la dinámica conjunta de comportamiento de dichas series. La predominancia de la especificación TGARCH sugiere que las buenas y malas noticias tienen impactos asimétricos sobre la volatilidad de los rendimientos. La predominancia de la distribución $t$ de Student multivariada sugiere que las perturbaciones no se distribuyen normalmente. 


\begin{tabular}{|c|c|c|c|c|c|c|c|c|c|}
\hline & \multicolumn{4}{|c|}{ Log likelihood } & \multicolumn{4}{|c|}{ Criterio Akaike } & \multirow{2}{*}{$\begin{array}{l}\text { Modelo } \\
\text { con } \\
\text { Mejor } \\
\text { Bondad } \\
\text { de } \\
\text { Ajuste }\end{array}$} \\
\hline & DVECH & & $\mathrm{CCC}$ & DBEKK & DVECH & & $\mathrm{CCC}$ & DBEKK & \\
\hline & \multicolumn{9}{|c|}{2 de octubre de 2000 al 10 de octubre de 2010} \\
\hline $\operatorname{AR}(1)-\operatorname{GARCH}(1,1)$ & 41906.86 & $*$ & 41611.98 & 4.14E+04 & -27.71912 & $*$ & -27.53707 & -27.44 & $\mathrm{CCC}$ \\
\hline $\operatorname{AR}(1)-\operatorname{TGARCH}(1,1)$ & $4.20 \mathrm{E}+04$ & * & 41706.48 & 4.16E+04 & -27.78924 & $*$ & -27.59634 & -27.55 & $\mathrm{CCC}$ \\
\hline $\begin{array}{l}\text { AR }(1)-G A R C H(1,1)- \\
\text { t Student }\end{array}$ & 42651.4 & $*$ & 42385.00 & 42345.08 & -28.21152 & $*$ & -28.04834 & -28.03 & $\mathrm{CCC}$ \\
\hline \multirow[t]{2}{*}{$\begin{array}{l}\text { AR }(1)-\mathrm{TGARCH}(1,1)- \\
\text { t Student }\end{array}$} & 42530.58 & & 42421.06 & 42414.39 & -28.14409 & & -28.06892 & -28.07 & DVECH \\
\hline & \multicolumn{9}{|c|}{2 de octubre de 2000 al 9 de agosto de 2007} \\
\hline $\operatorname{AR}(1)-\operatorname{GARCH}(1,1)$ & 33184.09 & * & 32863.36 & $3.28 \mathrm{E}+04$ & -38.52397 & $*$ & -38.19948 & -38.19887 & $\mathrm{CCC}$ \\
\hline $\operatorname{AR}(1)-\operatorname{TGARCH}(1,1)$ & 33025.30 & $*$ & 32909.96 & $3.29 \mathrm{E}+04$ & -38.38685 & $*$ & -38.24559 & -38.26528 & $\mathrm{CCC}$ \\
\hline $\begin{array}{l}\text { AR }(1)-\operatorname{GARCH}(1,1)- \\
\text { t Student }\end{array}$ & 33693.90 & * & 33400.12 & 33462.69 & -39.1163 & $*$ & -38.8232 & -38.92746 & $\mathrm{CCC}$ \\
\hline \multirow[t]{2}{*}{$\begin{array}{l}\text { AR }(1)-\mathrm{TGARCH}(1,1)- \\
\text { t Student }\end{array}$} & 33558.50 & * & 33419.18 & 33486.66 & -39.0064 & $*$ & -38.83723 & -38.94722 & DBEKK \\
\hline & \multicolumn{9}{|c|}{10 de agosto de 2007 al 10 de octubre de 2012} \\
\hline $\operatorname{AR}(1)-\operatorname{GARCH}(1,1)$ & 36665.12 & $*$ & 37524.43 & $3.64 \mathrm{E}+04$ & -56.08479 & $*$ & -57.57605 & -56.01 & $\mathrm{CCC}$ \\
\hline $\operatorname{AR}(1)-\operatorname{TGARCH}(1,1)$ & 33886.31 & $*$ & 37587.47 & $3.68 \mathrm{E}+04$ & -51.9774 & $*$ & -57.65611 & -56.52 & $\mathrm{CCC}$ \\
\hline $\begin{array}{l}\text { AR }(1)-G A R C H(1,1)- \\
\text { t Student }\end{array}$ & 38211.31 & $*$ & 38277.4 & 37606.09 & -58.46201 & $*$ & -58.73292 & -57.80 & $\mathrm{CCC}$ \\
\hline $\begin{array}{l}\text { AR(1)-TGARCH(1,1)- } \\
\text { t Student }\end{array}$ & 38093.35 & $*$ & 38300.33 & 37745.45 & -58.44823 & $*$ & -58.75128 & -58.00 & $\mathrm{CCC}$ \\
\hline
\end{tabular}

Notas: El asterico (*) denota que los modelos no cumplen con la condición de que la matriz de covarianza es semidefinida positiva. El modelo con mejor bondad de ajuste se determina con base en los estimadores considerados.

\section{Conclusiones y discusión}

En esta investigación se ha estudiado la validez de la hipótesis de eficiencia (ЕMH) en el mercado bursátil mexicano y se han modelado las series de rendimientos bursátiles. La evaluación de la EMH, en su forma débil, se ha realizado mediante estadísticas descriptivas, pruebas de raíces unitarias y de correlación. Los ejercicios de modelación y análisis econométricos han utilizado doce modelos GARCH multivariados con fines de comparativos y de caracterización de las series durante periodos de calma e inestabilidad financiera. El estudio se 
ha sustentado en una muestra de series de precios diarios de veinte acciones y del IPC que abarca datos del 2 de octubre de 2000 al 10 de octubre de 2012.

Los resultados de la investigación sugieren que hay escasa evidencia para sustentar la EMH en el mercado bursátil mexicano. El análisis de estacionariedad sugiere que la hipótesis de eficiencia predomina, hasta cierto punto, en el mercado cuando se consideran únicamente las series de precios de los activos. Sin embargo, la evidencia también sugiere que la eficiencia del mercado ha tendido a disminuir desde el año 2007. Por otra parte, la evidencia sugiere que las series de rendimientos bursátiles tienden a manifestar curtosis excesivas, clusters de volatilidad, asimetrías, distribuciones no normales, volatilidades no constantes y movimientos conjuntos de medias y varianzas.

Los ejercicios de modelación y análisis econométricos de las series de rendimientos validan las conclusiones anteriores. Los mismos muestran que: 1) Las series no validan la hipótesis de eficiencia porque los rendimientos tienden a estar significativamente correlacionados; 2) los modelos de tipo CCC son los que, en principio, mejor describen la dinámica de los series; 3) las buenas y malas noticias tienen impactos asimétricos sobre la volatilidad de los rendimientos; 4) por tanto, la especificación TGARCH caracteriza de mejor manera a la volatilidad de las series; y 5) la distribución $t$ de Student multivariada describe de mejor manera a las perturbaciones de los modelos.

La consistencia de los resultados obtenidos bien puede servir para justificar el estudio de las causas de la persistente ineficiencia del mercado bursátil mexicano. Particularmente, es nuestra creencia que esta se explica, parcialmente, por el escaso desarrollo del mercado. Este subdesarrollo se manifiesta en varios hechos estilizados. Entre estos se incluyen: 1) el pequeño y volátil número de empresas que hacen transacciones; ${ }^{17}$ 2) la gran concentración de la bursatilización en un pequeño conjunto de acciones de empresas de gran tamaño ${ }^{18}$ y 3) la existencia de problemas de asimetría de información. ${ }^{19}$ El estudio de estos hechos bien podría complementar el análisis aquí desarrollado.

\footnotetext{
${ }^{17}$ El escaso número de empresas que cotizan en el mercado bursátil mexicano se puede apreciar de manera comparativa si se considera que, a fines de 2012 cotizaban 143 emisoras en México. Por comparación, en Brasil, cotizaban alrededor de 500.

${ }^{18}$ El Índice de Precios y Cotizaciones (IPC) incluye datos de treinta y cinco emisoras que cotizan en la Bolsa Mexicana de Valores. Adviértase que el número total de emisoras en el último año de estudio fue 143 .

${ }^{19}$ El mercado bursátil mexicano, como otros mercados emergentes, no se caracteriza por contar con sistemas que provean información de manera rápida y a costos bajos, tal como ocurre en los mercados desarrollados.
} 
Finalmente, debe enfatizarse que la investigación referida a los mercados bursátiles tiene amplias posibilidades de desarrollo. En las economías emergentes, la importancia de este tipo de investigación se vincula directamente con el desarrollo económico y financiero. Esto ocurre porque los mercados bursátiles permiten financiar empresas, administrar riesgos, canalizar ahorros y asignar recursos para la inversión pública y privada. En este contexto, el desarrollo de investigación bursátil podría contribuir a que estas tareas pudieran realizarse de mejor manera. Por tanto, la importancia de realizar nuevas investigaciones puede justificarse plenamente desde las perspectivas teórica y empírica.

\section{Apéndice: \\ Matrices de Varianza-Covarianza Condicional}

En este apéndice se describen las matrices de varianza-covarianza condicional, $H_{t}$, que definen a los modelos DVECH, DBEKK y CCC usados en esta investigación. Estas matrices son importantes porque definen la dinámica de las series con base en ciertos supuestos estadísticos. Estas matrices son postuladas para efectos de estimación econométrica en virtud de que las "verdaderas" matrices de varianza-covarianza condicional no son observables. Tradicionalmente, los modelos GARCH multivariados (MGARCH) se definen y se nombran con base en los supuestos adoptados sobre la matriz de varianza-covarianza condicional.

La matriz de varianza-covarianza condicional del modelo de DVECH propuesto por Bollerslev, Engle y Wooldridge (1988) se caracteriza por tener un número bajo de parámetros a estimar. Esta propiedad permite que el número de parámetros a estimar sea relativamente reducido y por tanto lo hace relativamente sencillo de estimar. La matriz, $H_{t}$, del modelo DVECH es la siguiente:

$H_{t}=\Omega+A \circ \varepsilon_{t-1} \varepsilon_{t-1}^{\prime}+B \circ H_{t-1}$

donde las matrices de coeficientes $\Omega, A$ y $B$ son matrices simétricas de $N \times N^{20}$ y donde "o" denota el producto Hadamard. ${ }^{21}$

\footnotetext{
${ }^{20}$ Las matrices $\Omega, A$ y $B$ incluyen, respectivamente, a los términos constantes, ARCH y GARCH de la matriz de covarianza condicional. Se definen como matrices indefinidas para efectos de estimación econométrica.

${ }^{21}$ El producto de Hadamard de dos matrices $m \times n$ A y B se denota por $A \circ B$ y es una matriz $m \times n$ cuyas entradas están dadas por $(A \circ B)=a_{i j} b_{i j}$.
} 
La matriz de varianza-covarianza condicional del modelo DBEKK propuesto por Engle y Kroner (1995) se caracteriza por ser siempre positiva semi-definida (PSD). Esta propiedad conlleva a que este modelo siempre pueda estimarse de manera adecuada. Esta propiedad es importante para efectos de estimación econométrica porque no todas las propuestas de matrices de varianza-covarianza existentes en la literatura especializada satisfacen esta condición. ${ }^{22} \mathrm{La}$ matriz, $H_{t}$, del modelo DBEKK es la siguiente:

$H_{t}=\Omega \Omega^{\prime}+A \varepsilon_{t-1} \varepsilon_{t-1}^{\prime} A^{\prime}+B H_{t-1} B^{\prime}$

donde las matrices de coeficientes $\Omega, A$ y $B$ son matrices diagonales $N \times N$.

La matriz de varianza-covarianza del modelo CCC propuesto por Bollerslev (1990) no es diagonal. Aquí, las correlaciones condicionales son constantes en el tiempo y las covarianzas condicionales son proporcionales al producto de las desviaciones estándar condicionales correspondientes. Así, los elementos de la matriz,$H_{t}$, del modelo ccc son definidos como:

$h_{i i t}=c_{i}+a_{i} \varepsilon_{i t-1}^{2}+d_{i} I_{i t-1}^{-} \varepsilon_{i t-1}^{2}+b_{i} h_{i t t-1}$

$h_{i j t}=\rho_{i j} \sqrt{h_{i i t} h_{j j t}}$

donde $c_{i} a_{i} d_{i} b_{i}$ son constantes y donde $h_{i i t}, h_{i j t} \mathrm{y} \rho_{i j}$ son, respectivamente, la varianza, covarianza y coeficiente de correlación condicionales.

${ }_{22}$ Bauwens, Laurent y Rombouts (2006) detallan las condiciones que deben satisfacer las estimaciones de las matrices de covarianza condicional para que las mismas sean válidas econométricamente. 


\section{Referencias}

Aragonés, José R., y Juan Mascareñas, "La eficiencia y el equilibrio en los mercados de capital”, Análisis Financiero, 1994, Núm. 64, pp. 76-89.

Bachelier, Louis, Théorie de la Speculation, Tesis de Doctorado en Ciencias Matemáticas, Paris, Universidad Paris-Sorbona, 1900 [Traducción al inglés de D. May, 2011].

Ball, Ray, "The Global Financial Crisis and the Efficient Market Hypothesis: What we have learned?", Journal of Applied Corporate Finance, 2009, 21(4), otoño, pp. 8-16.

Bauwens, Luc, Sébastien Laurent, y Jeroen V. K. Rombouts, "Multivariate GARCH models: A survey", Journal of Applied Econometrics, 2006, 21(1), enero-febrero, pp. 79-109.

Bollerslev, Tim, "Generalized autoregressive conditional heteroskedasticity", Journal of Econometrics, 1986, 31(3), abril, pp. 307-327.

Bollerslev, Tim, "Modeling the coherence in short-run nominal exchange rates: A multivariate generalized ARCH model", Review of Economics and Statistics, 1990, 72(3), agosto, pp. 498-505.

Bollerslev, Tim, Robert F. Engle y Jeffrey M. Wooldridge, "A capital asset pricing model with time-varying covariances", Journal of Political Economy, 1988, 96(1), febrero, pp. 116-131.

Cabrero, Rodrigo, Rodolfo, Cermeño y Fausto Hernández-Trillo, "Eficiencia en el mercado accionario: Nueva evidencia para el caso mexicano”, Revista Mexicana de Economía y Finanzas, 2013, 8(1), enero-junio, pp. 53-74.

Cortés, José M. y Teresa Corzo, "La eficiencia en los mercados financieros: Una introducción a la cuestión”, Revista Empresa y Humanismo, 2009, 12(2), segundo semestre, pp. 81-106.

Duarte, Juan B. y Juan Mascareñas, "La eficiencia de los mercados de valores: Una revisión”, Análisis Financiero, 2013, Núm. 122, segundo cuatrimestre, pp. 21-35.

Durán-Vázquez, Rocio, Arturo Lorenzo-Valdés y Antonio Ruíz-Porras, "Valuation of Latin-American stock prices with alternative versions of the Ohlson model: An investigation of cointegration relationships with time-series and panel-data”, en Rafael Espinosa-Ramirez, (ed.), Research Issues in International Economic Relations, Universidad de Guadalajara- Editorial Universitaria, Zapopan, 2012, pp. 161-183.

Engle, Robert F., "Autoregressive conditional heteroscedasticity with estimates of the variance of United Kingdom inflation", Econometrica, 1982, 50(4), julio, pp. 987-1007. 
Economía Informa núm. 390 enero - febrero • 2015

Engle, Robert F. y Kenneth F. Kroner, "Multivariate simultaneous generalized ARCH”, Econometric Theory, 1995, 11(1), febrero, pp. 122-150.

Engle, Robert F., "GARCH 101: The use of ARCH/GARCH models in applied econometrics", Journal of Economic Perspectives, 2001, 15(4), otoño, pp. 157-168.

Engle, Robert F., Sergio M. Focardi, y Frank J. Fabozzi, “ARCH/GARCH models in applied financial econometrics”, en Frank J. Fabozzi (ed.), Handbook of Finance Vol. 3: Valuation, Financial Modeling and Qualitative Tools, John Wiley \& Sons, Hoboken, 2008, pp. 689-700.

Fama, Eugene F., "Random walks in stock market prices", Financial Analyst Journal, 1965, 21(5), septiembre-octubre, pp. 55-59.

Fama, Eugene F., "Efficient capital markets: A review of theory and empirical work", Journal of Finance, 1970, 25(2), mayo, pp. 383-417.

Fama, Eugene F., "Efficient capital markets II", Journal of Finance, 1991, 46(5), diciembre, pp. 1575-1617.

Hamilton, James D., Time Series Analysis, Nueva Jersey, Princeton University Press, 1994.

Hyme, Pauline, "La teoría de los mercados de capitales eficientes. Un examen crítico", Cuadernos de Economía, 2003, 22(39), julio-diciembre, pp. 57-83.

Levine, Ross y Sara Zervos, "Stock market development and long-run growth", The World Bank Economic Review, 1996, 10(2), pp. 323-339.

Ludlow, Jorge y Beatriz Mota, "Volatilidad del IPC, NASDAQ, y S\&P500: Un modelo GARCH multivariado", Análisis Económico, 2006, 21(48), tercer trimestre, pp. 215227.

López-Herrera, Francisco, "Análisis de la eficiencia del mercado accionario mexicano", Contaduría y Administración, 1998, (191), mayo-agosto, pp. 75-83.

López-Herrera, Francisco, Edgar Ortíz y Alejandra Cabello, "Las interrelaciones de volatilidad y rendimientos entre los mercados de valores del TLCAN", Investigación Económica, 2009, 68(267), enero-marzo, pp. 83-114.

López-Herrera, Francisco y Domingo Rodríguez-Benavides, "El efecto enero en las principales bolsas latinoamericanas de valores", Contaduría y Administración, 2010, (230), enero-abril, pp. 25-46.

López-Herrera, Francisco, Francisco Venegas-Martínez y César Gurrola-Ríos, “EMBI+ y su relación dinámica con otros factores de riesgo sistemático: 19972011”, Estudios Económicos, 2013, 28(2), julio-diciembre, pp. 193-216.

Lorenzo-Valdés, Arturo, Rocío Durán-Vázquez, y Leticia Armenta-Fraire, "Conditional correlation between oil and stock market returns: The case of Mexico", Revista Mexicana de Economía y Finanzas, 2012, 7(1), enero-junio, pp. 49-63. 
Lorenzo-Valdés, Arturo, y Antonio Ruiz-Porras, "Modelación de los rendimientos bursátiles mexicanos mediante los modelos TGARCH y EGARCH: Un estudio econométrico para 30 acciones y el Índice de Precios y Cotizaciones”, En Semei L. Coronado y L.A. Gatica, (eds.) Métodos no Lineales en Series Económicas y/o Financieras, Universidad de Guadalajara- Editorial Universitaria, Zapopan, 2012, pp. $46-81$.

Malkiel, Burton G., "The efficient market hypothesis and its critics", Journal of Economic Perspectives, 2003, 17(1), invierno, pp. 59-82.

Malkiel, Burton G., "Reflections on the efficient market hypothesis: 30 years later", The Financial Review, 2005, 40 (1), febrero, pp. 1-9.

Mandelbrot, Benoit, "Forecasts of future prices, unbiased markets, and 'martingale' models", Journal of Business, 1966, 39(1), enero, pp. 242-255.

Minovic, Jelena Z., "Modeling multivariate volatility procesess: Theory and evidence", Theoretical and Applied Economics, 2009, 5(5), mayo, pp. 21-44.

Samuelson, Paul A., "Proof that properly anticipated prices fluctuate randomly", Industrial Management Review, 1965, 6(2), primavera, pp. 41-49.

Santillán-Salgado, Roberto J., "Is the Mexican stock market becoming more efficient?", Revista Mexicana de Economía y Finanzas, 2011, 6(1), enero-junio, pp. 87-102.

Timmermann, Allan y Clive W.J. Granger, "Efficient market hypothesis and forecasting", , 2004, 20(1), enero-marzo, pp. 15-27.

Uribe-Gil, Jorge M., y Inés M. Ulloa-Villegas, "International Journal of Forecasting Revisando la hipótesis de los mercados eficientes: Nuevos datos, nuevas crisis y nuevas estimaciones", Cuadernos de Economía, 2011, 30(55), segundo semestre, pp. 127-154.

Yen, Gili, y Cheng-few Lee, "Efficient market hypothesis (ЕмH): Past, present and future", Review of Pacific Basin Financial Markets and Policies, 2008, 11(2), junio, pp. 305-329.

Zakoian, Jean-Michel, "Threshold heteroskedastic models", Journal of Economic Dynamics and Control, 1994, 8(5), septiembre, pp. 931-955. 